\title{
Retroperitoneal Hemorrhage During Veno-Venous Extracorporeal Membrane Oxygenation in COVID-19 Patientss: Clinical Experience and Review of Literature
}

\section{Jingchen Zhang}

Zhejiang University

\section{Xujian He}

Zhejiang University

Jia Hu

Zhejiang University

Tong Li ( $\boldsymbol{\sim}$ drli@zju.edu.cn )

Zhejiang University https://orcid.org/0000-0003-2011-1894

\section{Case report}

Keywords: Retroperitoneal Hemorrhage, ECMO, Anticoagulation, Prone Position Ventilation, Rehabilitation

Posted Date: June 5th, 2020

DOI: https://doi.org/10.21203/rs.3.rs-32685/v1

License: (c) (i) This work is licensed under a Creative Commons Attribution 4.0 International License.

Read Full License 


\section{Abstract}

Background: Retroperitoneal hemorrhage is a rare and severe complication in patients undergoing extracorporeal membrane oxygenation (ECMO). Retroperitoneal hematoma after the operation of multiple ECMO in a single center at the same time is extremely rare. The causes and treatment options can provide clinical experience and bring some inspiration.

Case presentation: Three cases of retroperitoneal hemorrhage patients with Corona Virus Disease-19 (COVID-19) are introduced; they had respiratory failure and were treated with veno-venous ECMO or venoarterial-venous ECMO. Retroperitoneal hemorrhage occurred during ECMO treatment. Among the three cases, 2 cases were found due to abdominal pain, and 1 case was found because of a decrease in ECMO circuit flow rate and hemoglobin level. 2 cases were treated with transcatheter arterial embolization, and 1 case was treated conservatively. The hemorrhage in each of the 3 cases did not deteriorate. Through early diagnosis and treatment, satisfactory treatment results were achieved for these 3 patients.

Conclusions: During the period of ECMO treatment, there is a low incidence of retroperitoneal hematoma, yet it comes with a high risk. This is due to anticoagulant use and some local mechanical injuries. If a decline in blood flow velocity and hemoglobin is detected, retroperitoneal hematoma should be taken into consideration, and early aggressive therapy should be started.

\section{Background}

Extracorporeal membrane oxygenation (ECMO) is a technology that can provide gas exchange and support cardiac function in patients with ARDS or cardiac failure ${ }^{1}$. Since the CESAR trial in 2009, venovenous ECMO has been established and widely used for the treatment of severe ARDS ${ }^{2,3}$. However, as an important salvage therapy, ECMO is harder to perform and maintain. One of the challenges of ECMO management is to achieve and maintain proper anticoagulation. In clinical practice, some factors, such as anticoagulation therapy and thrombocytopenia, can lead to bleeding complications. Common bleeding includes intubation-related bleeding, delayed gastrointestinal bleeding, and airway bleeding, which are typical clinical manifestations ${ }^{4}$.

Currently, Corona Virus Disease-19 (COVID-19) is spreading worldwide, with mortality rates in some countries as high as $10 \%$. Until now, there has been no specific treatment for COVID-19, and most of the deaths are caused by respiratory failure ${ }^{5}$. The use of ECMO in COVID-19 patients with severe respiratory failure is currently more controversial ${ }^{6,7}$. In our center, 10 COVID-19 patients used ECMO, and 7 of them had ECMO removed successfully and were recovering. Three cases of COVID-19 patients who experienced retroperitoneal hemorrhage during ECMO are reported in this paper. A review of the literature on similar presentations is provided, and our experience in treatment is also summarized.

\section{Case Presentation}


This study was approved by the Human Ethics Committee of the First Affiliated Hospital Zhejiang University. Informed written consent was obtained from the patients for publication of this case report and the accompanying images.

\section{Case 1}

A 71-year-old male underwent salvage ECMO with the following specifications on the 10th day after COVID-19 identification: mode: veno-venous; location of intubation: right femoral vein and right internal jugular vein; size of tubes: 19f, 22f; flow: $60 \mathrm{ml} / \mathrm{kg}$; gas: flow 0.8-1:1; and heparinization: initial bolus venous injection heparin dosage $25 \mathrm{U} / \mathrm{kg}$, and during the running time, an objective activated partial thromboplastin time (APTT) of 40-60 sec was maintained. His clinical course is shown in Fig. 1. On the 4th day of ECMO use, the assessment was passed, tracheal intubation was replaced with awake ECMO, and rehabilitation exercise was added. The training method involved sitting daily and passive lower limb functional training. After 8 days of ECMO use, the patient complained of abdominal pain, and his blood test showed that his hemoglobin $(\mathrm{Hb})$ was $60 \mathrm{~g} / \mathrm{l}$, his hematocrit was $26.2 \%$, and his lactic acid was $18 \mathrm{mmol} / \mathrm{l}$, accompanied by low ECMO circuit flow rates. Abdominal CT showed a retroperitoneal hemorrhage of $10.4 \times 8.6 \times 13.2 \mathrm{~cm}$ (Fig. 2 case 1). Red blood cells (14 units) and fresh-frozen plasma (10 units) were transfused, and anticoagulation was stopped. DSA showed bleeding in the right lumbar artery (Fig. 3A1), and transcatheter embolization was given to treat it (Fig. 3A2). Ultrasound was performed regularly to check the hemorrhage, and no deterioration was found.

\section{Case 2}

An 81-year-old female underwent salvage ECMO with the following specifications after 28 days of confirmed COVID-19: mode: veno-venous; location of intubation: left femoral vein and right internal jugular vein; size of tubes: 19f, 22f; flow: $60 \mathrm{ml} / \mathrm{kg}$; gas: flow 1:1; and heparinization: initial bolus venous injection heparin dosage $25 \mathrm{U} / \mathrm{kg}$, and during the running time, an objective APTT of 40-60 sec was maintained. Due to heart failure with veno-venous ECMO, veno-arterial-venous ECMO was used on the second to sixth day. The left femoral artery tube was 17F. Using a vascular stapler, the arterial end was closed, and the operation mode was changed to veno-venous -ECMO. The patient's sedation was maintained at Richmond Agitation Sedation Scale (RASS) -2 to RASS - 3. His clinical course is shown in Fig. 1. After 9 days of ECMO, a low ECMO circuit flow rate appeared, accompanied by a decrease in $\mathrm{Hb}$. A retroperitoneal hemorrhage was found on CT: $10.6 \times 10.3 \times 17.3 \mathrm{~cm}$ (Fig. 2 case 2$)$. Red blood cells (6 units) and fresh-frozen plasma (6 units) were transfused, and anticoagulation was stopped. DSA results showed that the lower right lumbar had an arterial hemorrhage (Fig. 3B1), and transcatheter arterial embolization (TAE) was given to cure it (Fig. 3B2). Ultrasound was performed regularly to check the hemorrhage, and significant changes were not discovered.

\section{Case 3}

A 62-year-old male underwent salvage ECMO with the following specifications after 31 days of confirmed COVID-19: mode: veno-venous; location of intubation: right femoral vein and right internal jugular vein; 
size of tubes: 19f, 22f; flow: $60 \mathrm{ml} / \mathrm{kg}$; gas: flow 1:1; and heparinization: initial bolus venous injection heparin dosage $50 \mathrm{U} / \mathrm{kg}$, and during the running time, an objective APTT of 40-60 sec was maintained. Digestive tract bleeding occurred during ECMO for 13 days, and rectal cancer was diagnosed. Under the support of ECMO, radical rectal cancer surgery was performed. After 11 days of ECMO, mechanical ventilation was withdrawn and switched to awake ECMO. The rehabilitation exercises of the patient involved sitting on the bedside, combined with electric bicycles and limb rehabilitation exercises. His clinical course is shown in Fig. 1. The patient complained of abdominal pain after 18 days of ECMO. His vital signs were as follows: blood pressure dropped to $85-100 / 50-65 \mathrm{mmHg}$; heart rate was $110-135$ b.p.m. Ultrasound showed edema of the psoas muscle, and CT showed a retroperitoneal hemorrhage of $8.2 \times 4.2 \times 6.5 \mathrm{~cm}$ (Fig. 2 case 3 ). Anticoagulation was stopped, rehabilitation out of bed was suspended, and the hemorrhage was stable.

\section{Discussion And Conclusions}

To minimize thromboembolic complications and maintain patency of the circuit, proper anticoagulation is essential during ECMO. Bleeding complications are one of the most common problems in elderly patients who undergo ECMO, and they are also an important cause of accidental death. In this study, three patients had retroperitoneal hemorrhage beyond 7 days after ECMO initiation. There were no related trauma or iatrogenic procedures. Current risk factors for idiopathic retroperitoneal hemorrhage include anticoagulation, antiplatelet therapy, and chronic renal failure ${ }^{8}$.

Retroperitoneal bleeding itself is difficult to diagnose and can present with nonspecific symptoms. The literature indicates that $10.1 \%$ of retroperitoneal hemorrhages are misdiagnosed or never diagnosed 4 . Ranney D, et al pointed out that retroperitoneal hemorrhages should be considered when ECMO fluctuates or ECMO circuit flow rates are low despite volume infusion ${ }^{9}$. During ECMO, bleeding situations, such as bleeding at the puncture site and gastrointestinal bleeding, can easily cover a retroperitoneal hemorrhage. At present, CT is the first recommendation for diagnosing retroperitoneal hemorrhage, and ultrasound is only accepted as an evaluation criterion ${ }^{10}$. Three patients in this study were diagnosed with retroperitoneal hemorrhage by $\mathrm{CT}$ and routinely monitored by ultrasound.

The three patients with COVID-19 in this study were elderly patients who underwent ECMO for severe ARDS. Low platelet levels $\left(<5010^{3} / \mu \mathrm{L}\right)$ during ECMO have become one of the reasons for the formation of retroperitoneal hemorrhage. Among them, the patient in Case 3 had experienced a replacement of an ECMO oxygenator before the retroperitoneal hemorrhage. This operation gave rise to a transient consumption of coagulation substances. During the first 4 hours after oxygenator replacement: APTT $78.7 \mathrm{~s}$; prothrombin time intertional normalized ratio (PT-INR): 1.2 ; and platelets $94^{\star} 10^{9} / \mathrm{L}$. This may cause bleeding, which should cause alarm.

In addition to antithrombotic therapy, other causes of retroperitoneal hemorrhage include trauma and benign and malignant renal tumors ${ }^{11}$. Three cases of retroperitoneal hemorrhage in this report showed multiple diffuse bleeding, and physical shock should be considered first. The course of COVID-19 was 
long, and protective clothing increased the difficulty of caretaking for the nursing staff. Inadequate care of the body position, particularly the waist and back, and the frequent prone position for the treatment of patients and imaging examinations can cause long-term compression of the waist and back and affect blood circulation.

Another possible mechanism is bleeding from rehabilitation injuries because two of the patients had bleeding after rehabilitation. Therefore, specific programs should be developed for elderly patients with ECMO to reduce the incidence of retroperitoneal hemorrhage. At present, there are similar reports of retroperitoneal hemorrhage during ECMO, but the etiology and early diagnosis are less discussed ${ }^{12}$. Three cases were reported in our center at one time, and the pathogenesis and possible causes were analyzed. These three patients received care and active support in the intensive care unit (ICU), including blood transfusions, coagulopathy reversal, and radiation interventions. The patients in case 1 and case 2 underwent TAE hemostasis within 2 hours; the patient in case 3 was treated with corrected blood coagulation to reduce postural injury and recover from injury. If patients have a significant decrease in hemoglobin and exhibit shock, early TAE treatment is recommended.

Retroperitoneal hemorrhages in patients with ECMO often have severe consequences. Some suggestions are as follows: elderly patients need a combination of awake ECMO and rehabilitation; patients should use anticoagulation reduction standards; changes in blood coagulation function and hemoglobin should be closely monitored; routine ultrasound examination should be performed to determine the occurrence of retroperitoneal hemorrhages; if ECMO patients undergo a transfer, a patient transfer board and other aided measures are recommended; and if patients complain of lower back pain, with decreased $\mathrm{Hb}$, or decreased ECMO flow, retroperitoneal bleeding should be considered.

\section{Declarations}

\section{Ethics approval and consent to participate}

This study was approved by the Human Ethics Committee of the First Affiliated Hospital Zhejiang university. The patient gave consent for publication of this case report and images.

\section{Consent for publication}

Written informed consent was obtained from the patient for publication of this case report and accompanying images.

\section{Availability of data and materials}

The datasets used during the current study are available from the corresponding author on reasonable request. 


\section{Competing interests}

The authors have declared no competing interests.

\section{Funding}

None.

\section{Authors' Contributions}

JC Zhang generated the idea of writing the case report and was the consultant in charge of the patient. $\mathrm{JC}$ Zhang and XJ He reviewed the case notes of the patient and wrote the original draft of the case presentation. J Hu and T Li significantly revised the original draft and added the conclusions and references. All authors contributed to the final version of the manuscript.

\section{Acknowledgments}

The authors would like to thank all participants of the study, the nurses and clinical staff who are providing care for the patients.

\section{References}

1. None. What is ECMO? American Journal of Respiratory \& Critical Care Medicine, 2016, 193(6): P9P10.

2. Peek GJ, Mugford M, Tiruvoipati R et al. Efficacy and economic assessment of conventional ventilatory support versus extracorporeal membrane oxygenation for severe adult respiratory failure (CESAR): a multicentre randomised controlled trial. Lancet 2009; 374: 1351-63.

3. Noah MA, Peek GJ, Finney SJ et al. Referral to an extracorporeal membrane oxygenation center and mortality among patients with severe 2009 influenza A(H1N1). JAMA 2011; 306: 1659-68.

4. Sunga K L, Bellolio M F, Gilmore R M, et al. Spontaneous Retroperitoneal Hematoma: Etiology, Characteristics, Management, and Outcome[J]. The Journal of Emergency Medicine, 2012, 43(2): 157-e161.

5. Lai CC, Shih TP, Ko WC, Tang HJ, Hsueh PR. Severe acute respiratory syndrome coronavirus 2 (SARSCoV-2) and coronavirus disease-2019 (COVID-19): The epidemic and the challenges. Int J Antimicrob Agents. 2020;55(3):105924.

6. Henry BM. COVID-19, ECMO, and lymphopenia: a word of caution [published online ahead of print, 2020 Mar 13]. Lancet Respir Med. 2020; 2213-2600(20)30119-3.

7. MacLaren G, Fisher D, Brodie D. Preparing for the Most Critically III Patients With COVID- 
8. Hwang N K, Rhee H, Kim I Y, et al. Three cases of spontaneous lumbar artery rupture in hemodialysis patients[J]. Hemodialysis International, 2016.

9. Ranney D, Hatch S, Bonadonna D, et al. ECMO Flow as a Sign of Intraabdominal Hemorrhage After Prolonged CPR[J]. ASAIO Journal, 2018.

10. Zissin R, Ellis M, Gayer G. The CT Findings of Abdominal Anticoagulant-Related Hematomas[J]. Seminars in Ultrasound Ct \& Mr, 2006, 27(2):0-125.

11. Ibrahim W, Mohamed A, Sheikh M, et al. Antiplatelet Therapy and Spontaneous Retroperitoneal Hematoma: A Case Report and Literature Review[J]. American Journal of Case Reports, 2017, 18:8589.

12. Sugiura G, Bunya N, Yamaoka A, et al. Delayed retroperitoneal hemorrhage during veno-venous extracorporeal membrane oxygenation: a case report[J]. Acute Medicine \& Surgery, 2019, 6(2):180184.

\section{Figures}
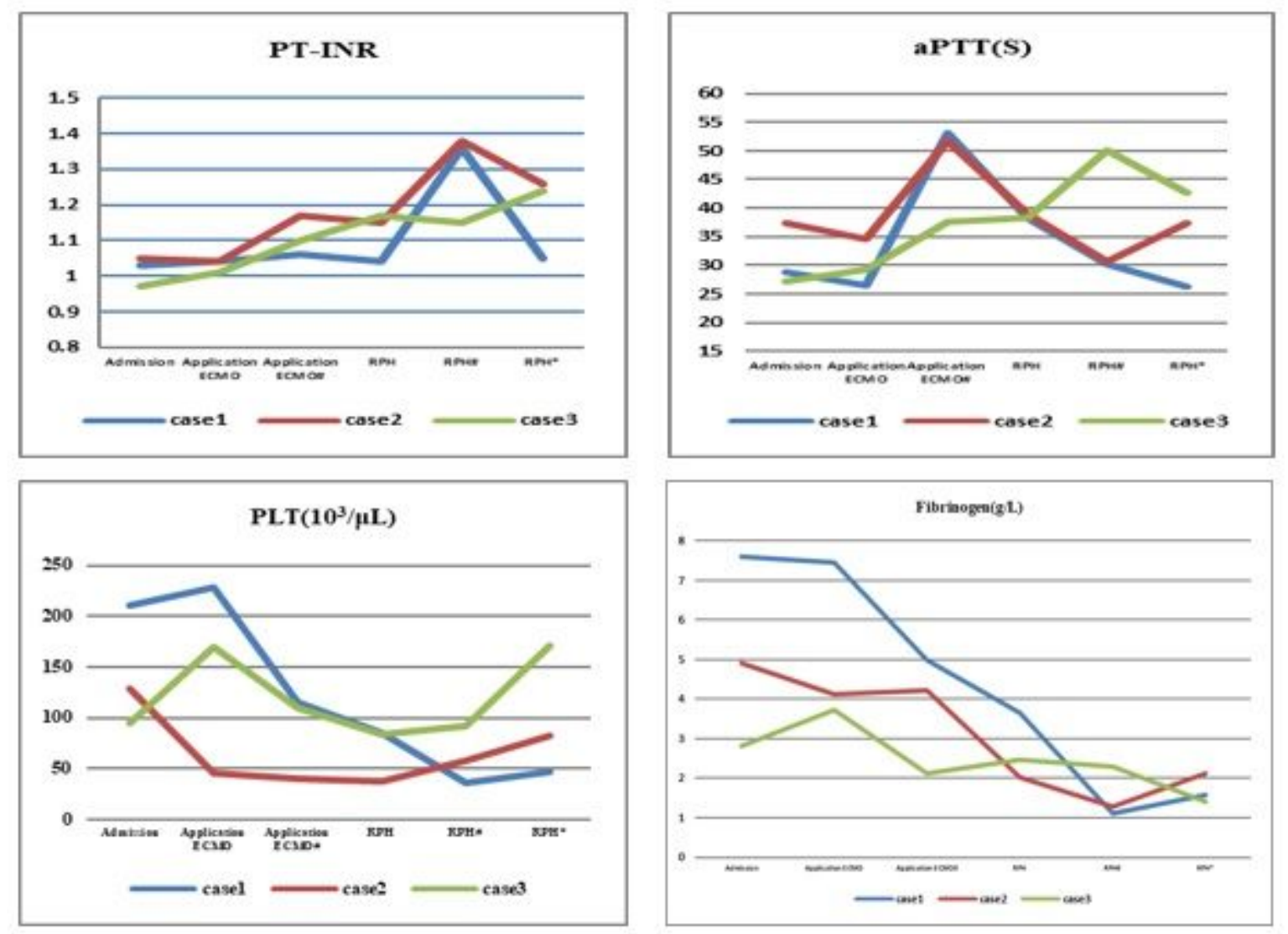

\section{Figure 1}

Three patients suffer from delayed retroperitoneal hemorrhage during veno-venous extracorporeal membrane oxygenation (ECMO) treatment. PT-INR, PLT, and Fibrinogen have obvious changes before and after RPH, which is consistent with bleeding symptom, while APTT has no typical signs because 
anticoagulation is used during the ECMO treatment. Abbreviation $\triangle \mathrm{PT}-\mathrm{INR}$ : Prothrombin Time Intertional Normalized Ratio; PLT: Platelets \#second day *third day
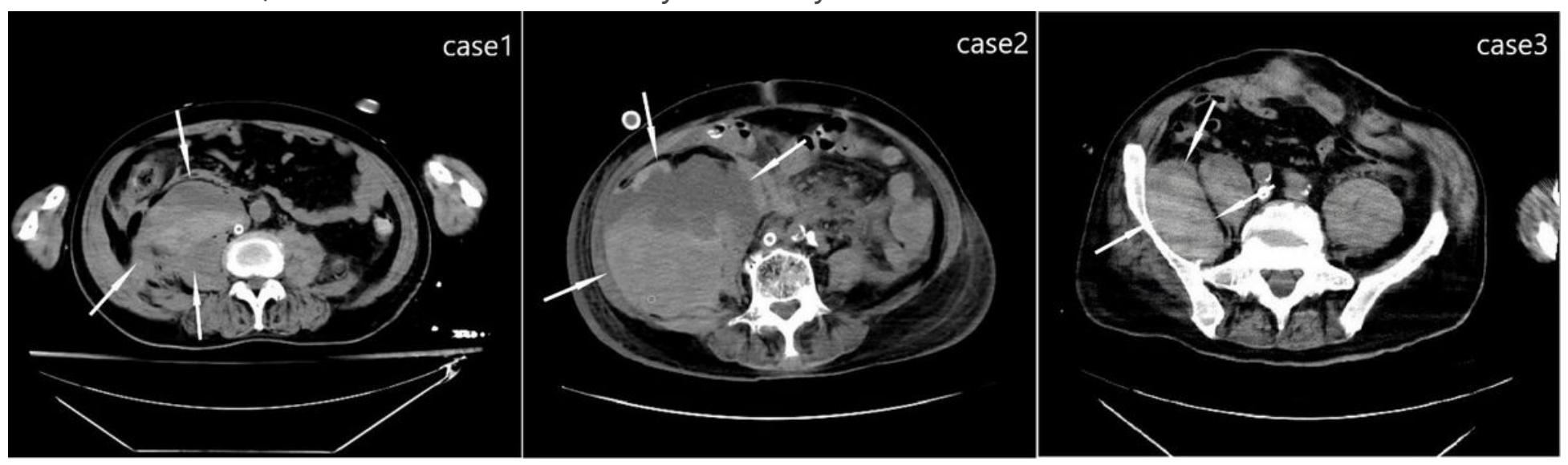

\section{Figure 2}

Three patient retroperitoneal hematoma (arrow) CT scan 

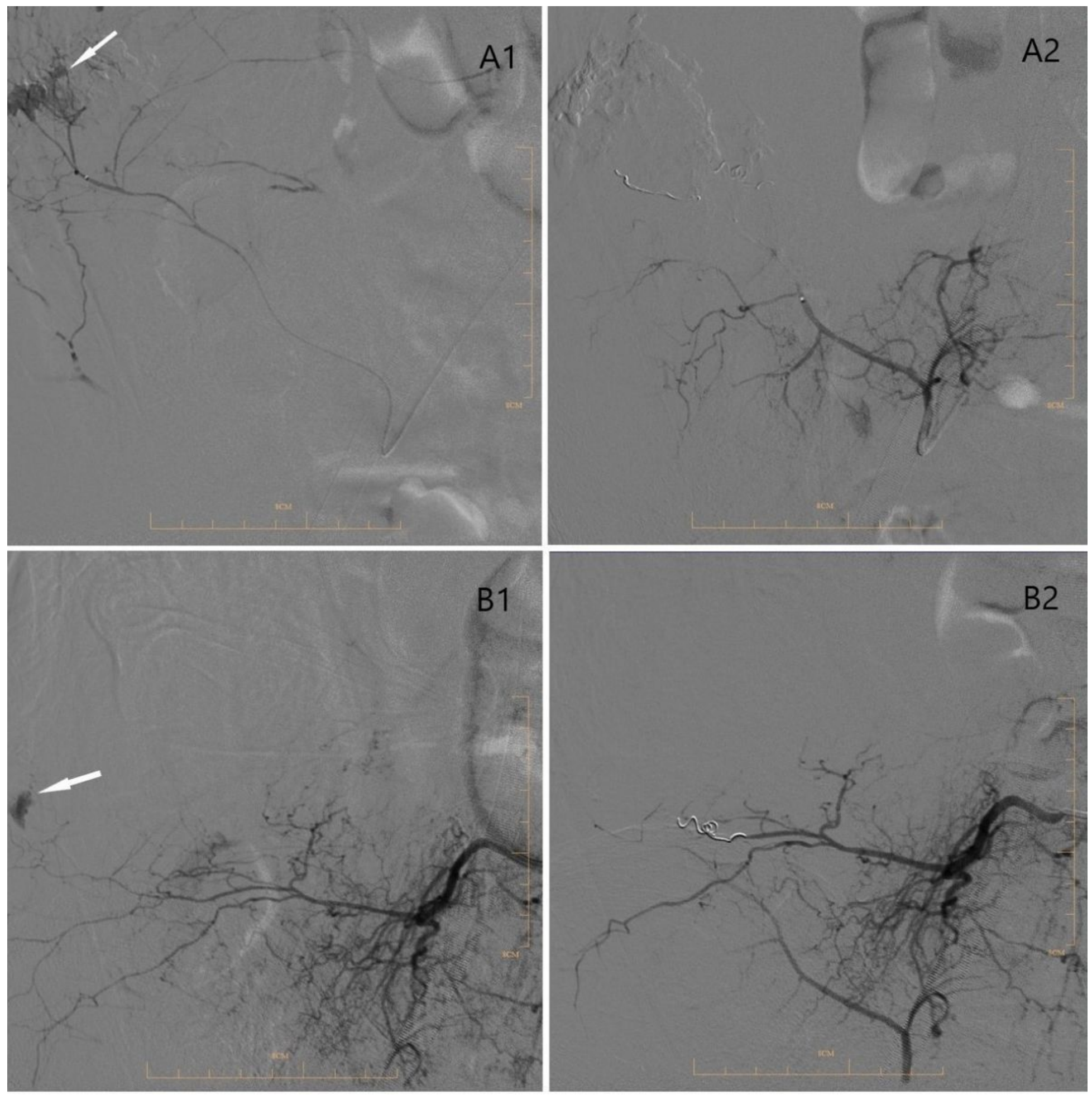

\section{Figure 3}

$A 1$ is case 1. The DSA in A1 shows the effusion of hematomas》arrow \in arteriae lumbales; $A 2$ refers to the vascular image after the TAE treatment; $B 1$ is case 2. The DSA in B1 gives the information about the effusion of hematomas】arrow \in the internal iliac artery branch; B2 stands for the vascular image after the TAE treatment. 